\title{
Vermittlung der Abtönungspartikeln im DaF-Unterricht
}

\section{Modal particles in language education}

\begin{abstract}
This paper is an attempt to present German modal particles in language education. The author describes the theoretical basis of teaching of modal particles on different levels and formulates the main problems of teaching and learning modals particles.
\end{abstract}

KEYWORDS: modal particles, language education.

Abtönungspartikeln stellen nicht nur hinsichtlich ihrer Grammatikographie, sondern auch unter translatorischem, didaktischem und lexikographischem Aspekt ein interessantes Forschungsgebiet dar. Auch unter dem Aspekt des Deutschen als Fremdsprache sind die Ergebnisse der Partikelforschung von erheblicher Bedeutung, nicht zuletzt für die Konzipierung von auf mündliche bzw. dialogische Kommunikationskompetenz ausgerichteten Lehrwerken. Das Ziel des vorliegenden Aufsatzes ist die Darstellung der Lehr- und Lernprobleme, die sich aus dem Erwerb der Abtönungspartikeln durch Ausländer ergeben können.

Nach Helbig/Kötz (1981: 7ff.) werden Abtönungspartikeln zu den schwierigsten Aspekten der deutschen Sprache im DaF-Unterricht gezählt, mit denen sogar die weit Fortgeschrittenen Schwierigkeiten haben. Daher erweist sich auch ihre Didaktik als problematisch. Helbig/Kötz verweisen darauf, dass die Beschreibung der Partikeln (i.S.v Abtönungspartikeln) unter dem didaktischen Aspekt notwendig ist. Diese Notwendigkeit begründen die Autoren durch die Tatsache, dass Partikeln im Deutschen im Gegensatz zu anderen Sprachen hochfrequent sind. Ihre Frequenz zeichnet sich vor 
allem in der Alltagssprache und Dialogen aus, d.h. in der auf das Mündliche ausgerichteten Sprache (Helbig/Kötz 1981: 7). Der Haupterscheinungsbereich der Abtönungspartikeln ist somit die Umgangssprache. Im Gegensatz zur genormten Schriftsprache umfasst die (gesprochene) Umgangssprache auch Mundartliches. Zu ergänzen ist hier, dass der Partikelreichtum und -gebrauch unabhängig von der sozialen Stellung des Sprechers ist. Im Allgemeinen lässt sich feststellen: je umgangssprachlicher, desto abtönungsreicher (vgl. Weydt 1969: 96).

Weydt (1991: 60) verweist darauf, dass u.a. folgende Fragen bezüglich der Vermittlung von Abtönungspartikeln im Deutschunterricht im Vordergrund stehen sollen:

- Wie wichtig ist es für Ausländer, Abtönungspartikeln zu beherrschen und wie beurteilen die Deutschen Ausländer, die Abtönungspartikeln oder Partikeln im Allgemeinen gut beherrschen und solche, die sie nicht verwenden können?

- Was sind die Schwierigkeiten, die Ausländer mit dem Erwerben der Abtönungspartikeln haben?

- Wie kann man den Erwerbsprozess in Bezug auf Abtönungspartikeln steuern und fördern?

- Welcher Übungs- und Lehrverfahren können sich Lehrer im Deutschunterricht bedienen?

Die Antwort auf die erste Frage versucht Busse (1992: 32) zu geben, indem er Folgendes feststellt:

Partikellose Sprache ist im Deutschen eindeutig als barsch, schroff oder apodiktisch markiert; wenn ausländische Deutschlerner oftmals ihre Verwunderung darüber kundtun, dass der Kommunikationsstil der Deutschen „zu sachlich“, krass unverbindlich oder gar unhöflich sein, so ist dies ein Hinweis darauf, dass sie in ihrem Fremdsprachenunterricht den Partikelgebrauch nicht gelernt haben und ihnen damit die wichtigsten kommunikativen Möglichkeiten fehlen, welche die Partikeln den Deutschen bereitstellen. Das Lehren von Partikeln ist aber trotz der Probleme der Bedeutungsbeschreibung, die ja auch Probleme der Bedeutungsvermittlung und damit der Didaktik sind, unbedingt notwendig, wenn die Deutschlernenden ein nicht als schroff oder wenigstens eindeutig unidiomatisch markiertes Deutsch lernen sollen (mit allen negativen Folgen, die dies im sozialen Verkehr haben kann).

Fraglich mag sein, ob die deutsche Spreche ohne Abtönungspartikeln tatsächlich apodiktisch wirkt. Außer Zweifel steht aber, dass Abtönungspartikeln eine wichtige pragmatische Funktion zukommt, die mit keinen anderen lexikalischen Mitteln (außer mit außersprachlichen Mitteln wie Mimik und Gestik) verglichen werden kann. 
Die zweite von Weydt gestellte Frage betrifft die Schwierigkeiten, die mit dem Erwerben der Abtönungspartikeln verbunden sind. Diese Schwierigkeiten sind vor allem auf die zahlreichen Funktionen der Abtönungspartikeln zurückzuführen, denn es lässt sich nicht immer bestimmen, welche Funktion die gegebene Abtönungspartikel in einem Satz hat und wie sie zu verstehen ist (vgl. Frączyk 2002: 787). Außerdem haben Abtönungspartikeln homonyme Entsprechungen in anderen Wortklassen, was sich bei ihrer Vermittlung und im Erwerbsprozess als problematisch erweisen kann.

Abtönungspartikeln scheinen einfach weglassbar zu sein. Nach Kemme (1979: 3) würde aber ein Ausländer, der das Deutsche erlernt, ohne sich die Abtönungspartikeln anzueignen, „ein sehr untypisches Deutsch erwerben“. Deswegen müssen Abtönungspartikeln in Lehrwerke eingeführt werden, und zwar so früh wie möglich. Sie erscheinen dort meistens in dialogischen Texten, die reale Redesituationen imitieren. Sie werden aber meistens in separaten Übungen nicht erklärt. Als Grund für diesen Tatbestand nennt Kemme die Tatsache, dass sich die Abtönungspartikeln selbst für einen deutschen Muttersprachler, Deutschlehrer oder Lehrbuchautor schwer erklären lassen. Der zweite Grund dafür ist, dass sich der Entwurf von Übungen im Bereich Abtönungspartikeln als problematisch und schwierig erweist. Im Weiteren wir darauf hingewiesen, dass die meisten Schwierigkeiten beim Erwerb der deutschen Abtönungspartikeln durch Ausländer u.a. auf folgende Faktoren zurückzuführen sind (vgl. Kemme 1979: 3f.):

- manche Lernende sperren sich gegen imitatives und intuitives Lernen,

- der Lernende lernt intuitiv, verlangt jedoch ausdrückliche Erklärungen, die im Falle der Abtönungspartikeln nicht vorhanden sind,

- Lehrer können bestimmte Fragen bezüglich der Abtönungspartikeln nicht erklären und besprechen,

- Lernende haben nicht genügend Gelegenheiten zum Einüben und Befestigen der Abtönungspartikeln, was auf Fehler in Übungen zu ihnen zurückzuführen ist,

- Sie werden oft mit ihren homonymen Entsprechungen aus anderen Wortklassen verwechselt.

Interessanterweise weist Kemme (1979: 3f.) auch darauf hin, dass die gegebene Muttersprache - laut einer Studie des Goethe-Instituts - keinen Einfluss auf den Erwerb der Abtönungspartikeln bei Ausländern habe. Daher kann davon ausgegangen werden, dass der Erwerbsprozess bei allen Lernern gleich erfolgt, ungeachtet dessen, ob in der gegebenen Ausgangssprache funktional ähnliche Wörter vorliegen oder nicht. Nach dieser Annahme kann festgehalten werden, dass sich der Erwerb und das Verständnis der Abtönungspartikeln auch bei den polnischen Deutschlernenden als problematisch und schwierig erweist, und das obwohl das Polnische über Abtö- 
nungspartikeln verfügt, derer Funktionen sich zu einem guten Teil mit ihren deutschen Entsprechungen überdecken.

Außer Zweifel steht, dass die Lehrwerke für Deutsch als Fremdsprache auf das Kommunikationsvermögen ausgerichtet sein sollten. Abtönungspartikeln lassen sich am einfachsten in gesprochene dialogische Texte einbringen, und nur solche Texte kommen der gesprochenen Umgangssprache nahe. In der Tat bilden jedoch Dialoge nur einen kleinen Teil der Lehrwerke, weswegen zusätzliche Übungen zur Verwendung der Abtönungspartikeln wünschenswert sind.

Es wird empfohlen, in allen Grundstufen Abtönungspartikeln zu üben, jedoch nach der jeweiligen Stufe entsprechenden methodischen Ansätzen. Nach Kemme (1979: 21ff.) sollten in der Grundstufe bestimmte und typische Verwendungsweisen und Funktionsvarianten einer Partikel geübt werden, vor allem durch das Wiederholen typischer Sätze und Muster, die zu Modellen werden. So ist der Lernende im Stande, unbewusst Regeln zu bilden und sie intuitiv zu verwenden. Der Prozess solcher innerlichen Modellbildung kann durch gezielte Übungen beschleunigt werden. Kemme betont, dass bei solcher Art der Übungen der Kontext mitberücksichtigt werden sollte. In dieser Phase soll auf keine zusätzlichen Fragen, wie etwa homonyme Entsprechungen oder verschiedene Funktionsvarianten eines Partikellexems eingegangen werden. Erst später können spezifische Erklärungen zu den Abtönungspartikeln angegeben werden. Zu der Erklärung der Abtönungspartikeln schreibt Kemme (1979: 23):

Modalpartikeln (i.S.v. Abtönungspartikeln - J.D.-A.) sind aber schwer zu erklären. Eindeutige, kurze Regeln, von denen sich Äußerungen mit Modalpartikeln schlüssig ableiten lassen, sind kaum zu erstellen. Hier sollte in jedem Fall der intuitive Lernprozeß (der über Hypothesenbildung natürlich auch kognitiv ist) jeder ausdrücklichen Erklärung vorangehen. Wieweit die Erklärung danach eine Rolle spielt, muß sich nach dem Interesse der Schüler richten.

Die grammatische Beschreibung von Abtönungspartikeln kann nach Kemme erst von der Mittelstufe an erfolgen.

Im Weiteren verweisen Helbig/Kötz (1981: 43f.) darauf, dass die Frage der Abtönungspartikeln in vielen Lehrmaterialien zu wenig berücksichtigt wird oder gar keine speziellen Lehrmaterialien vorliegen. Dafür führen die Autoren folgende Gründe an:

- Auch ohne Beherrschung der Partikeln ist dem Ausländer eine erfolgreiche Kommunikation in der deutschen Sprache möglich. Allerdings kann er dann manche Nuancen nicht verstehen und schon gar nicht ausdrücken. (...) 
- Die ungenügende methodische Aufbereitung der Behandlung der Partikeln ergibt sich auch daraus, dass die linguistischen Vorgaben zu unvollständig sind (...). Das gilt weniger für die Beschreibung unter semantischem und syntaktischem Gesichtspunkt, sehr stark aber für die Beschreibung der Partikeln unter dem Gesichtspunkt ihrer kommunikativen Funktion.

- Die ungenügende methodische Aufbereitung der Behandlung der Partikeln erklärt sich wohl auch daraus, daß die Frage noch ungeklärt ist, inwieweit Partikeln lehr- und übbar sind. (...)

- Es bestanden und bestehen in der Linguistik sehr unterschiedliche Auffassungen zu den Partikeln (...). Auch von hier aus ergeben sich Schwierigkeiten für ihre methodische Aufbereitung.

Im Gegensatz zu Kemme, der der Meinung ist, dass das Erwerben der Abtönungspartikeln in der Grundstufe durch das gezielte Wiederholen von Mustern erfolgen soll, vertreten Helbig, Kötz (1981) die Auffassung, dass sie wie andere Vokabeln vermittelt werden sollen. Sie schlagen eine glossarische Behandlung der Abtönungspartikeln vor, aber nur mit der einen Bedeutung, die eine Abtönungspartikel an einer bestimmten Stelle realisiert (vgl. Helbig, Kötz 1981: 46). Die von Kemme vorgeschlagene Methode hat den Vorteil, dass durch die gezielte Bildung der Satzmodelle, in denen eine Partikel verwendet werden kann, tatsächlich der intuitive Lernprozess gefördert wird. Die von Helbig, Kötz vorgeschlagene Methode, d.h. das glossarische Erwerben der Abtönungspartikeln kann dazu führen, dass der Lernende lediglich die eine Entsprechung einer Abtönungspartikel erwerben wird. Das bringt die Gefahr mit, dass die gegebene Äußerung nicht verstanden wird, wenn eine Abtönungspartikel in einem anderen Kontext mit einer anderen Funktionsschattierung erscheint. Für die Mittelstufe schlagen Helbig/Kötz vor, Abtönungspartikeln weiter glossarisch zu behandeln. Im Weiteren soll in dieser Stufe den Lernenden bewusst gemacht werden, welche Rolle Partikeln in der deutschen Grammatikographie spielen. Dazu können die Lehrenden auf das Problem der Homonyme von Abtönungspartikeln in anderen Wortklassen eingehen (z.B. die Primärfunktion von aber als Konjunktor und aber als Abtönungspartikel). Eine systematische theoretisch fundierte Behandlung der Partikeln bleibt jedoch nur für die Fortgeschrittenenstufe vorbehalten (vgl. Helbig, Kötz 1981: 46).

Die präsentierten Auffassungen lassen sich in folgenden methodischen Hinweisen zusammenfassen:

- Das Erwerben der Abtönungspartikeln soll sowohl rezeptiv als auch produktiv erfolgen. Ratsam ist daher das gezielte Wiederholen von Mustersätzen, um eine intuitive Verwendung der Abtönungspartikeln durch die Lerner zu erreichen. 
- Besonders hochfrequentative Partikeln sollten produktiv gebraucht werden, während weniger gebräuchliche nur rezeptiv vermittelt werden können (vgl. Helbig, Kötz 1981: 47).

- Ab der Mittelstufe kann bei der Vermittlung der Abtönungspartikeln auf ihre homonymen Entsprechungen eingegangen werden.

- Die systematische Behandlung der Partikeln insgesamt erfolgt erst auf der Fortgeschrittenenstufe.

- Das Übungsmaterial sollte sich auf die gesprochene und möglichst wirklichkeitsnahe Situationen stützen.

Den oben dargestellten Auffassungen ist gemein, dass eine genaue grammatische Beschreibung der Abtönungspartikeln erst ab der fortgeschrittenen Stufe vorgeschlagen wird. Diese Beschreibung kann meines Erachtens nach dem Satzartkriterium erfolgen. In Bezug auf die Satzarten und die Einbringung von Abtönungspartikeln in diese schreibt Helbig (1998: 126):

Es handelt sich [...] um eine starke, aber nicht absolute Korrelation zwischen SA und Partikelsektion [...]. Gewiß gibt es Partikeln, die für die einzelne SA charakteristisch sind.

Helbig betont daher, dass es möglich und legitim ist, Abtönungspartikeln und deren Funktionsschattierungen, anhand des Satzartkriteriums zu beschreiben. Diesen Ansatz kann man m.E. auch unter didaktischem Aspekt anwenden. Der umgekehrte Weg, Satzarten mittels Abtönungspartikeln zu differenzieren, ist indessen weder zuverlässig noch methodologisch begründet (vgl. Helbig 1998: 126). Frączyk (2002: 783) schreibt zu dem Zusammenhang zwischen Satzarten und Abtönungspartikeln:

Mit der Wahl eines bestimmten Satztyps entscheidet sich der Sprecher für eine bestimmte Modalität. Entweder will er etwas erfahren, bezweifeln, jemanden informieren oder zu etwas veranlassen. Da der Sprecher nicht alle Informationen explizit ausdrücken kann, verwendet er die Partikeln [i.S.v. Abtönungspartikeln - J.D.-A.], um einen Hintergrund für die Interpretation des Satzes zu schaffen. Mit der Verwendung der Partikel instruiert er den Hörer, wie er eine Aussage verstehen soll, und veranlasst ihn zu einer Stellungnahme. Der Rezipient kann mit der Äußerung entweder einverstanden sein oder ihr widersprechen, auf jeden Fall muss er aktiv sein, auch wenn er es sprachlich nicht ausdrückt.

Es bleibt also zu erwägen, die Frage der Satzartendistribution im DaFUnterricht zu klären. Dadurch wird den Lernenden bewusst, in welchen Satzarten die gegebene Abtönungspartikel eingebracht werden kann, was zum Verständnis der pragmatischen Funktion der Abtönungspartikeln wesentlich beitragen kann. Im Folgenden werden die Distributionsbeschränkungen der Abtönungspartikeln nach den vier Basissatzarten dargestellt: 
1. Konstativsätze (mit Aussage- und Ausrufeintention)

aber 1

Du kommst aber pünktlich!

auch $_{1}$

Ich habe von dem Text nicht alles verstanden - Na ja, Französisch ist auch nicht einfach.

bloß 1

Das ist bloß eine Vermutung!

doch $_{1}$

Darüber haben wir doch schon gesprochen.

eben $_{1}$

Das ist eben so.

eigentlich ${ }_{1}$

Das ist eigentlich ganz richtig.

einfach $_{1}$

Ich kann es einfach nicht tun.

etwa $\mathbf{a}_{1}$

Sie ist doch nicht etwa krank.

halt $_{1}$

Das Spiel haben wir halt verloren.

ja $\mathbf{1}_{1}$

Das ist ja eine Überraschung!

(ein) mal $_{1}$

Ich erkläre dir mal später.

ruhig $_{1}$

Du kannst ihn ruhig fragen.

schon $_{1}$ (unbetont)

Sie werden schon pünktlich kommen.

vielleicht

Das war vielleicht spannend!

wohl 1

Sie hat wohl den Zug verpasst.

2. Entscheidungsfragesätze (mit Frage- und Ausrufeintention)

aber $_{2}$

Bist du aber groß geworden!

auch $_{2}$

Hast du dich auch gut vorbereitet?

denn $_{1}$

Hast du das denn gesehen?

eigentlich ${ }_{2}$

Warst du eigentlich schon einmal in Russland?

einfach $_{2}$

Kannst du ihm einfach nicht die Wahrheit sagen? etwa 2

Willst du schon etwa gehen? 
mal $_{2}$

Kannst du mir mal helfen?

nicht

Ist das Wetter nicht herrlich?

vielleicht $_{2}$

Soll ich darauf vielleicht verzichten? wohl $_{2}$

Wirst du wohl sofort verschwinden!

3. Ergänzungsfragesätze (mit Frage- und Ausrufe-Intention oder implizierter Antwort)

aber $_{3}$

Was war das aber für ein Abend!

auch $_{3}$

Ich bin heute sehr müde. - Warum gehst du auch immer so spät ins Bett?

bitte $_{1}$

Was soll das bitte heißen?

bloß 2

Warum ist er bloß nicht gekommen?

denn $_{2}$

Was gibt es denn Neues? (als Frage)

Was ist denn hier los? (als Vorwurf)

$\operatorname{doch}_{2}$

Wie heißt doch deine Tochter?

eigentlich $_{3}$

Wo ist er eigentlich?

einfach $_{3}$

Wie konntest du es einfach tun?

nur $_{1}$

Wie spät ist es nur?

schon $_{2}$

Wer will schon in einer Baracke wohnen?

wohl $_{3}$

Warum sind sie wohl gekommen?

\section{Aufforderungssätze}

bitte $_{2}$

Geh doch bitte zum Arzt!

bloß $\aleph_{3}$

Geh mir bloß aus dem Weg!

$\operatorname{doch}_{3}$

Setz dich doch!

eben $_{2} /$ halt $_{2}$

Mir ist zu war. - Zieh dich eben/halt leichter an!

einfach $_{4}$

Gehen Sie einfach zum Arzt! 
$\mathbf{j a}_{2}$

$$
\begin{aligned}
& \text { Sei ja pünktlich! } \\
& \text { mal }_{3}
\end{aligned}
$$

Komm mal her!

nur $_{2}$

Lass mich nur in Ruhe lesen!

ruhig $_{2}$

schon $_{3}$

Kommt ruhig herein!

Beeile dich schon!

Als hilfreich bei der Vermittlung der Abtönungspartikeln im DaF-Unterricht kann sich auch die Frage ihrer Homonyme in anderen Wortklassen erweisen, worauf bereits hingewiesen wurde. Die Einführung und der Vergleich der Homonyme der Abtönungspartikeln im Deutschen ermöglicht deren klare Abgrenzung und kann so Verwechslungen vorbeugen. Im Folgenden sind die Homonyme der Abtönungspartikeln zusammengestellt:

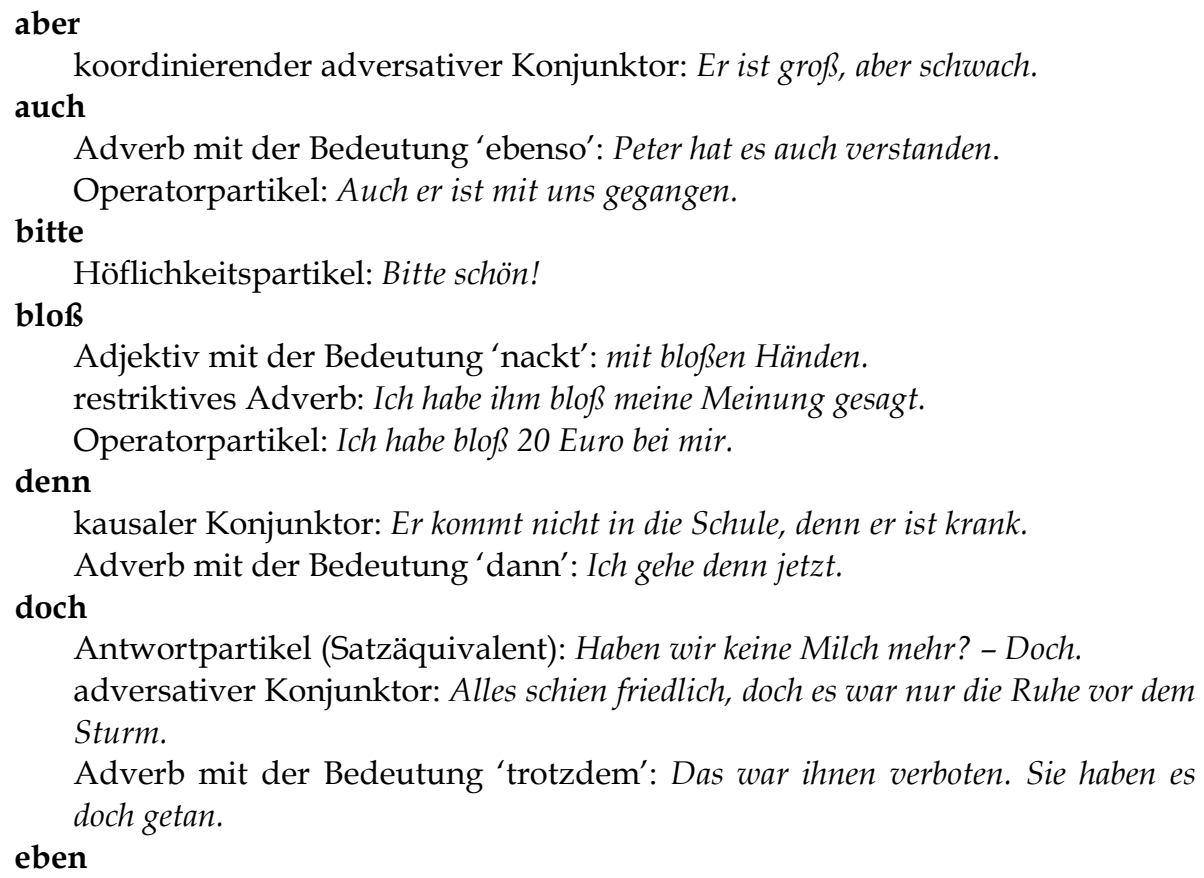

Adverb mit der Bedeutung 'ebenso': Peter hat es auch verstanden.

Operatorpartikel: Auch er ist mit uns gegangen.

\section{bitte}

Höflichkeitspartikel: Bitte schön!

\section{bloß}

Adjektiv mit der Bedeutung 'nackt': mit bloßen Händen.

restriktives Adverb: Ich habe ihm bloß meine Meinung gesagt.

Operatorpartikel: Ich habe bloß 20 Euro bei mir.

\section{denn}

kausaler Konjunktor: Er kommt nicht in die Schule, denn er ist krank.

Adverb mit der Bedeutung 'dann': Ich gehe denn jetzt. doch

Antwortpartikel (Satzäquivalent): Haben wir keine Milch mehr? - Doch.

adversativer Konjunktor: Alles schien friedlich, doch es war nur die Ruhe vor dem Sturm.

Adverb mit der Bedeutung 'trotzdem': Das war ihnen verboten. Sie haben es eben doch getan.

Adjektiv mit der Bedeutung 'flach' oder 'gleichmäßig': eine ebene Landschaft.

Temporaladverb: Der Zug ist eben angekommen.

Operatorpartikel: Eben diese Frau habe ich gestern am Tatort gesehen.

eigentlich

Adjektiv: der eigentliche Grund. 
einfach

Adjektiv: Die Aufgabe war viel einfacher als ich erwartet habe. etwa

Operatorpartikel: Vor dem Ministerium gab es etwa 100 Demonstranten. halt

Imperativ von halten: halt!

Deverbativum: der Halt. ja

Antwortpartikel (Satzäquivalent): Ist er zu Hause? - Ja.

Operatorpartikel mit der Bedeutung 'sogar': Das ist eine sehr gute Arbeit, ja hervorragend!

mal / Mal

(ugs.) Wiederholungszahladverb: Warst du schon mal in Italien?

Nomen: Sei gegrüßt viel tausend Mal ...

nicht

Negationspartikel: Ich habe ihn gestern nicht gesehen.

nur

restriktives Adverb: Die Stelle schien interessant, nur die Entfernung war zu groß.

Operatorpartikel (vergleichbar mir bloß): Es kamen nur 10 Personen zum Treffen. ruhig

Adjektiv mit der Bedeutung 'friedlich' oder 'ungestört': Die See war ruhig. schon

Adverb: Das Kind schläft ruhig.

Temporaladverb mit der Bedeutung 'bereits': Ich bin schon da.

Operatorpartikel: Es ist schon sehr spät.

vielleicht

Satzadverb: Vielleicht kommt er schon morgen.

wohl

Adverb: Ich fühle mich heute nicht wohl.

Abschließend möchte ich festhalten, dass der Fähigkeit, eine Fremdsprache in Alltagsituationen und alltäglichen Gesprächen $\mathrm{zu}$ verwenden, im Fremdsprachenunterricht besonderes Augenmerk zukommen sollte. Dazu gehört auch das Verständnis und die korrekte Verwendung der deutschen Abtönungspartikeln. Diese sicher sowohl für Lernende als auch und Lehrende nicht einfache Aufgabe kann mit entsprechenden Übungen gelöst werden, u.a. durch solche, die die Satzartdistribution und die Homonyme der Partikeln berücksichtigen.

\section{LITERATURVERZEICHNIS}

Busse, D., 1992. Partikeln im Unterricht Deutsch als Fremdsprache. In: Muttersprache 102, 37-59. Frączyk, D., 2002. Einige Bemerkungen zum Status der Abtönungspartikel aus kontrastiver Sicht: deutsch-polnisch. In: Studia Niemcoznawcze XXIV, 779-790. 
Helbig, G., 1998. Plädoyer für Satzarten. In: Donhauser, K., Eichinger, L. M. (Hrsg.). Deutsche Grammatik - Thema in Variationen. Heidelberg: Universitätsverlag Winter, 121-136.

Helbig, G., Kötz, W., 1981. Die Partikeln. Leipzig: VEB Verlag Enzyklopädie.

Kemme, H., 1979. "Ja", "denn", "doch", usw. Die Modalpartikeln im Deutschen. Erklärungen und Übung für den Unterricht an Ausländer. München: Goethe-Institut.

Weydt, H., 1969. Abtönungspartikeln. Die deutschen Modalwörter und ihre französischen Entsprechungen. Bad Homburg: Gehlen Verlag.

Weydt, H., 1981. Methoden und Fragestellungen der Partikelforschung. In: Weydt, H. (Hrsg.). Partikeln im Deutschunterricht. Heidelberg: Groos Verlag, 45-63. 